\title{
Plant Selenium Hyperaccumulation Affects Rhizosphere: Enhanced Species Richness and Altered Species Composition
}

\begin{abstract}
Alyssa T. Cochran and Jemma Bauer, Department of Biology, Colorado State University, Fort Collins 80523; Jessica L. Metcalf, Department of Ecology and Evolutionary Biology, CU Boulder, Boulder, CO 80309, and Department of Animal Sciences, Colorado State University, Fort Collins 80523; Petra Lovecka and Martina Sura de Jong, Department of Biochemistry and Microbiology, University of Chemistry and Technology, 166 28, Prague, Czech Republic; Sven Warris, Paul J. W. Mooijman, and Ingrid van der Meer, Bioscience, Wageningen University and Research, 6708 PB 4, Wageningen, The Netherlands; Rob Knight, Departments of Pediatrics and Computer Science \& Engineering, and Center for Microbiome Innovation, University of California at San Diego, La Jolla 90203; and Elizabeth A. H. Pilon-Smits, ${ }^{\dagger}$ Department of Biology, Colorado State University, Fort Collins 80523
\end{abstract}

Accepted for publication 11 May 2018.

\section{ABSTRACT}

Little is known about the microbiomes associated with plants with unusual properties, including plants that hyperaccumulate toxic elements such as selenium (Se). Se hyperaccumulators contain up to $1.5 \%$ of their dry weight in Se, concentrations shown to affect ecological interactions with herbivores, fungal pathogens and neighboring plants. Hyperaccumulators also enrich their surrounding soil with Se, which may alter the rhizobiome. To investigate whether plant Se affects rhizobacterial diversity and composition, we used a combination of culture-independent and culture-based approaches. Sequencing of 16S rRNA gene amplicons using the Illumina platform revealed that the rhizosphere microbiomes of Se hyperaccumulators were significantly different from nonaccumulators from the same site, with a higher average relative abundance of Pedobacter and Deviosa. Additionally, hyperaccumulators harbored a higher rhizobacterial species richness when compared with nonaccumulators from the same family on the same site. Independent from Se present at the site or in the host plant, the bacterial isolates were extremely resistant to selenate and selenite (up to $200 \mathrm{mM}$ ) and could reduce selenite to elemental Se. In conclusion, Se hyperaccumulation does not appear to negatively affect rhizobacterial diversity, and may select for certain taxa in the rhizosphere microbiome. Additionally, Se resistance in hyperaccumulator-associated bacteria and archaea may be widespread and not under selection by the host plant.

Additional keyword: phyllosphere.
The rise of next-generation sequencing technologies has made microbiome research an increasingly popular and feasible area of study in plant biology (Berg et al. 2013). These technologies have allowed researchers to study entire plant-associated microbial assemblages in addition to microbes that require culture-dependent

${ }^{\dagger}$ Corresponding author: E. A. H. Pilon-Smits; E-mail: epsmits@ colostate.edu

Funding: This project was made possible by funding from Colorado State University, the Stavros Family Fund, and the Earth Microbiome Project.

*The $e$-Xtra logo stands for "electronic extra" and indicates that two supplementary tables are published online.

Current address of P. Lovecka and M. Sura de Jong: Life Sciences and Technologies, Van Hall Larenstein University of Applied Sciences, 8901 BV, Leeuwarden, The Netherlands.

(C) 2018 The American Phytopathological Society analyses. Additionally, next-generation sequencing technologies have allowed the exploration of core microbiomes: stable, consistent components across complex microbial assemblages. A core has been defined as the suite of members shared among microbial consortia from similar habitats (Shade and Handelsman 2011). Identification of a core microbiome for a certain habitat offers a starting point to further explore the functional role and importance of particular microbial taxa or consortia in the habitat in question. Microbiome sequencing has offered a new and more expanded view of the microbial world, which has changed the way researchers look at the microbial communities associated with plants (Berg and Hallmann 2006; Berg et al. 2013; Visioli et al. 2015).

An area of microbiome research that has received increasing interest is plant hyperaccumulation, i.e., the capacity of some plant species to accumulate upwards of 100-fold higher levels of a specific element than their nonaccumulator counterparts, to levels that are toxic to most plants (Baker and Brooks 1989; Cappa and 
Pilon-Smits 2014). Plants that are hyperaccumulators (HAs) represent a niche that may support and/or be shaped by selected consortia of microorganisms. The extreme concentration of a toxic element in or around HA may affect the plant's microbiome. Conversely, it is also possible that the microbiome affects elemental accumulation.

Around 500 plant species have been reported to be hyperaccumulators of one or more of the elements arsenic (As), cadmium $(\mathrm{Cd})$, cobalt $(\mathrm{Co})$, nickel $(\mathrm{Ni})$, lead $(\mathrm{Pb})$, selenium $(\mathrm{Se})$, and zinc (Zn) (Cappa and Pilon-Smits 2014). Only around 10\% of known HA species have been studied with respect to rhizosphere processes (Alford et al. 2010; Visioli et al. 2015). A recent microbiome sequencing study comparing $\mathrm{Cd} / \mathrm{Zn} \mathrm{HA}$ and non-HA genotypes of Sedum alfredii, Luo et al. (2017) found host genotype to explain a small, but significant amount of variation that may affect metal hyperaccumulation. Earlier, culture-based research has shown that the rhizosphere bacteria of $\mathrm{Ni}$ and $\mathrm{Cd} \mathrm{HAs}$ are more tolerant to the metals that their hosts hyperaccumulate than rhizosphere microbes not associated with HAs (Visioli et al. 2015). In addition, inoculation of HA-derived microbes back to their HA hosts (grown from surface-sterilized seed) in some cases enabled the plants to accumulate more of the element in question (Visioli et al. 2015). These results suggest a role for microbes in making these toxic elements available to HA plants.

The focus of the current study is the interaction between Se HA plants and their rhizosphere microbiomes, with the main aim to study the effects of Se HA on rhizosphere prokaryotic microbiome diversity and composition. Selenium is an essential micronutrient for many microbes and animals, including humans, and is considered a beneficial element for higher plants (Pilon-Smits et al. 2009). Despite not being essential for higher plants, Se is readily taken up by plants and assimilated from selenate or selenite to organic forms via the sulfate assimilation pathway (Schiavon and Pilon-Smits 2017). Selenium HAs are native to the Western United States where they often occur on naturally Se-rich (seleniferous) soils and can contain tissue Se levels up to $1.5 \%$ of dry weight (Beath et al. 1939). Selenium is toxic to most organisms at high concentrations because it is converted into selenocysteine (SeCys), which disrupts protein function when it is nonspecifically incorporated into proteins in the place of cysteine (Terry et al. 2000). Selenium HAs such as Stanleya pinnata (Brassicaceae) and Astragalus bisulcatus (Fabaceae) have evolved a way to circumvent this toxicity by converting SeCys into a nontoxic form, methylSeCys, which can be sequestered in epidermal vacuoles or further converted to volatile dimethyldiselenide (DMDSe) (Evans and Johnson 1967; Neuhierl et al. 1999). Many bacteria and fungi detoxify Se in a similar way, taking up selenate or selenite and converting it to organic forms or volatile forms; they can also reduce selenite or selenate to insoluble elemental $\mathrm{Se}\left(\mathrm{Se}^{0}\right)$ (Frankenberger and Karlson 1994; Staicu et al. 2015a,b; Turner et al. 1998; Winkel et al. 2015; Zayed and Terry 1994). Most bacteria have the capacity to convert selenite, but not selenate, to elemental Se, which has a deep red color and is less toxic than selenite (Garbisu et al. 1996). Many bacteria require Se as an essential element, while fungi appear to have lost the physiological requirement for Se (Zhang and Gladyshev 2009).

It is unclear how Se HA plants affect the diversity and composition of rhizosphere microbial consortia. Several lines of evidence suggest that high-Se substrates are actually more microbe-rich than corresponding low-Se substrates, including plant litter and ponds (de Souza et al. 2001; Quinn et al. 2011a). Even when highSe substrates are clearly selecting for taxa that are more Se-resistant, evidence exists for increased rhizosphere microbial diversity. For example, rhizosphere fungi isolated from naturally seleniferous soils were more tolerant to high concentrations of Se than those isolated from a nonseleniferous area, and their rhizosphere fungal communities were more diverse and compositionally distinct (Wangeline et al. 2011). It is not known whether the same is the case for rhizosphere bacteria and archaea. Bacterial endophytes from HAs were able to withstand (and in some cases, benefit from) selenate and selenite concentrations up to $200 \mathrm{mM}$ (Sura de Jong et al. 2015); the same may be true for rhizosphere bacteria. Specialized Se-resistant rhizosphere microbes may benefit their host. When inoculated to plants, both rhizosphere and endosphere microbes can promote plant growth, increase Se accumulation, and increase pathogen resistance (Alford et al. 2014; de Souza et al. 1999a,b; Di Gregorio et al. 2005; El Mehdawi et al. 2015; Hanson et al. 2003; Lindblom et al. 2012, 2013; Sura de Jong et al. 2015; Yasin et al. 2015). However, the effect of selection for Se bacterial resistance on the diversity and composition of the rhizosphere bacterial and archaeal diversity remains a knowledge gap.

In this study, a combination of culture-dependent and cultureindependent methods was used to study the rhizosphere microbiomes of plants that hyperaccumulate Se in comparison with non-HA species or unvegetated soil from the same seleniferous area or from a nearby nonseleniferous area. The aim of this study was to answer the following questions. (i) Is the Se HA rhizosphere microbiome different from related non-HA species in terms of composition and diversity? (ii) Are bacteria isolated from Se HAs or seleniferous soil more resistant to Se than those from non-HAs or nonseleniferous soil? (iii) Is there a core rhizomicrobiome among Se hyperaccumulators that may contribute to Se hyperaccumulation?

\section{MATERIALS AND METHODS}

Sampling. Rhizosphere soil was sampled from five plant species growing on a seleniferous site, Pine Ridge, in Fort Collins, CO

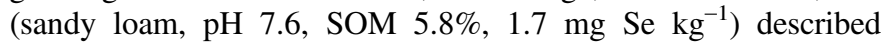
previously (El Mehdawi et al. 2012; Galeas et al. 2008). The sampled species include three HA species, Astragalus bisulcatus (Fabaceae) and Stanleya pinnata (Brassicaceae) and an HA population of Symphyotrichum ericoides (Asteraceae) and two nonHA species, Astragalus tenellus (Fabaceae) and Physaria bellii (Brassicaceae). For comparison, rhizosphere soil was sampled from plants on a nearby nonseleniferous site, Cloudy Pass, in Bellvue,

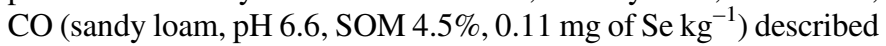
by El Mehdawi et al. (2012, 2015). The species sampled from Cloudy Pass were all non-HA species, including Astragalus laxmannii (Fabaceae), Physaria montana (Brassicaceae) and a non-Se accumulator population of Symphyotrichum ericoides described by El Mehdawi et al. (2015). Leaf and rhizosphere soil samples were taken from six individuals of each species sampled on both sites, except for A. tenellus $(n=4)$. Rhizosphere soil was collected by removing the root from the soil, shaking the bulk soil off of the roots, and then collecting remaining soil in association with the roots in a sterile container. Soil samples were then sieved using a $1 \mathrm{~mm}$ sieve and stored in sterile 1.5-ml Eppendorf tubes and stored at $-80^{\circ} \mathrm{C}$ before microbiome analysis. Leaf Se concentrations of the plants collected from Pine Ridge were evaluated using inductively coupled plasma optical emission spectrometry (ICP-OES) after drying and acid digestion, according to Fassel (1978) and as described earlier (Quinn et al. 2011a). The Cloudy Pass vegetation was found earlier to have negligible tissue Se concentration and was not tested here. Plant species and Se concentrations of the sampled plants are listed in Table 1.

Culture-independent studies: DNA extraction and 16S rRNA gene amplification for rhizosphere microbiome sequencing. Sample processing, sequencing, and core amplicon data analysis were performed by the Earth Microbiome Project (EMP) 
(www.earthmicrobiome.org) (Gilbert et al. 2014; Thompson et al. 2017), and all amplicon sequence data and metadata have been made public through the data portal (http://qiita.ucsd.edu) as QIITA study ID 10363 and EBI accession ERP017439.

Data processing and statistics of 16S rRNA gene sequences. Initial data processing of $16 \mathrm{~S}$ rRNA gene sequence data were provided by the EMP (Gilbert et al. 2014; Thompson et al. 2017) using QIIME version 1.9.1 (Caporaso et al. 2010) in QIITA following standard processing protocols. Closed reference operational taxonomic unit (OTU) picking was used against the greengenes 13_8 database (http://www.earthmicrobiome.org/protocols-and-standards/ initial-qiime-processing/). Briefly, $16 \mathrm{~S}$ rRNA gene sequences were filtered for quality and demultiplexed. OTUs representing chloroplast DNA were filtered out and the resulting table was rarefied at 45,750 sequences per sample. An unweighted UniFrac distance matrix was generated from OTUs detected in each sample and visualized using a principal coordinates analysis plot. Permanova and Anosim statistical tests (999 permutations) were performed on the UniFrac distance matrix using for the entire sample set as well as within the Pine Ridge sample set. The frequency of all OTUs were compared between sites and within Pine Ridge samples using a Kruskal Wallis test. Analysis of variance (ANOVA) was used in JMP, Version 11 (SAS Institute Inc., Cary, NC) to compare alpha diversity (observed OTUs) between sites and within Pine Ridge. Additionally, core microbiomes for Pine Ridge, Cloudy Pass, HAs, and non-HAs were computed using the QIIME core microbiome script 'compute_ core_microbiome.py' with the minimum fraction of samples that an OTU must be observed in to be considered part of the core set to 1.0.

Culture-based studies. Rhizosphere bacterial isolation. Rhizosphere soil samples were sieved with a $1 \mathrm{~mm}$ screen. A 1:1 (wt/vol) ratio of soil and autoclaved $50 \mathrm{mM} \mathrm{MgSO}_{4}$ were mixed and diluted to $10^{-6}$ before plating $200 \mu$ lonto Luria Bertani (LB) agar medium (peptone at $10 \mathrm{~g} \mathrm{liter}^{-1}$, yeast extract at $5 \mathrm{~g} \mathrm{liter}^{-1}$, and sodium chloride at $10 \mathrm{~g} \mathrm{liter}^{-1}$ ) and incubated at $30^{\circ} \mathrm{C}$ for 3 to 10 days. This medium is relatively rich, and like all media likely yielded colonies from only a subset of the bacteria present in the sample, in this case favoring fast-growing species. Individual colonies were isolated based on morphology and cultured in LB liquid before being stored in a 1:1 ( $\mathrm{vol} / \mathrm{vol})$ solution of bacterial culture and $30 \%$ glycerol at $-80^{\circ} \mathrm{C}$. Individual isolates were identified via matrixassisted laser desorption ionization-time of flight mass spectrometry (MALDI-TOF MS) using Autoflex Speed 93 MALDI-TOF/TOF mass spectrometer and MALDI Biotyper 3.1 software (Bruker Daltonik, 94 Germany) as described by Sura de Jong et al. (2015). A list of all bacterial strains and their identification confidence levels used in this study can be found in Supplementary Table S1.

Selenium resistance. Individual isolates were streaked onto LB plates containing $0,1,10,100$, or $200 \mathrm{mM}$ sodium selenate $\left(\mathrm{Na}_{2} \mathrm{SeO}_{4}\right)$ or sodium selenite $\left(\mathrm{Na}_{2} \mathrm{SeO}_{3}\right)$ and qualitatively scored for Se resistance as well as for the ability to produce red elemental Se. Each isolate that grew on the minus Se plate was then given a selenate and selenite resistance score ( 0 to 5$)$ based on the ability to grow on each plate. The scores were assigned based on the following criteria: 0 indicates no growth on any of the Se plates, 1 denotes growth up to $1 \mathrm{mM} \mathrm{Se}, 2$ indicates growth up to $10 \mathrm{mM}$ Se, 3 indicates growth up to $100 \mathrm{mM}, 4$ indicates growth up to $200 \mathrm{mM}$, and a score of 5 denotes improved growth at $200 \mathrm{mM} \mathrm{Se}$ (compared with the control plate).

Then, each of the selenate and selenite resistance scores were pooled for each individual plant host. The median selenate and selenite resistance scores were calculated for each host plant. Scores from each host plant were then put into the following three groups: (i) Cloudy Pass non-HA; (ii) Pine Ridge HA; and (iii) Pine Ridge non-HA; statistical comparisons of the qualitative Se tolerance scores in these groups were performed using a Kruskal-Wallis test with the software package JMP, Version 11 (SAS Institute Inc.).

\section{RESULTS}

Culture-independent methods. Next generation sequencing of $16 \mathrm{~S}$ rRNA gene amplicons indicated that the most prevalent bacterial phyla in the data set were Proteobacteria (23.3\%), Actinobacteria (17.8\%), Bacteroidetes (17.6\%), and Acidobacteria (16.4\%) (Fig. 1).

Permanova and Anosim tests of the UniFrac distance matrix revealed significant phylogenetic community differences between Pine Ridge and Cloudy Pass sample sites $(P<0.001)$ (Fig. 2). We also discovered significant differences $(P<0.001)$ in prokaryotic community composition between HA, non-HA and bulk soil over the whole data set. We then focused only on the Pine Ridge site and found significant differences in community composition between HA, non-HA and bulk soil $(P<0.001)$ (Fig. 3). When looking at Pine Ridge specifically, no significant differences were found in individual OTUs relative abundance when comparing HA soil with non-HA soil and bulk soil. While not statistically significant, it is noteworthy that two OTUs had a substantially higher mean occurrence in HA-associated rhizosphere soil than in other soil samples, that may warrant further study: Pedobacter and Devosia.

Alpha diversity (observed OTUs) was also tested between sites and within Pine Ridge. Between sites, Cloudy Pass had a significantly higher alpha diversity than Pine Ridge (ANOVA, $P<0.004$ ) (Fig. 4), with non-HA from Cloudy Pass having a similar alpha diversity as HA from Pine Ridge. Within Pine Ridge, however, samples from HA were found to be significantly more diverse than samples taken from non-HAs and bulk soil (ANOVA, $P<0.001$ ). Interestingly, the rhizosphere samples from HA species had a higher alpha diversity (Fig. 4). Similar results were obtained for phylogenetic distance based alpha diversity measures (not shown).

TABLE 1

Plant species sampled for this study ( $n=6$ plants per species), and corresponding leaf selenium (Se) range determined by inductively coupled plasma optical emission spectrometry (ICP-OES) ${ }^{\mathrm{a}}$

\begin{tabular}{|c|c|c|c|}
\hline Pine Ridge (seleniferous) & $\begin{array}{l}\text { Leaf Se concentration } \\
\left(\mathrm{mg} \text { of Se } \mathrm{kg}^{-1} \text { of } \mathrm{DW}\right)\end{array}$ & Cloudy Pass (nonseleniferous) & $\begin{array}{l}\text { Leaf Se concentration } \\
\left(\mathrm{mg} \text { of } \mathrm{Se} \mathrm{kg}^{-1} \text { of } \mathrm{DW}\right)\end{array}$ \\
\hline Astragalus bisulcatus (HA species) & $10-2,699$ & Astragalus laxmannii (non-HA species) & $0.07-0.11$ \\
\hline Physaria bellii (non-HA species) & $3-24$ & Physaria montana (non-HA species) & $0.02-0.35$ \\
\hline Symphyotrichum ericoides (HA population) & $36-1,169$ & Symphyotrichum ericoides (non-HA population) & ND \\
\hline
\end{tabular}

${ }^{a}$ Leaf, root, and rhizosphere soil samples were taken from each plant. HA, Se hyperaccumulator. ND = not determined. 
To identify possible key microbes that play a role in plant Se hyperaccumulation, core microbiomes (microbes identified in $100 \%$ of the samples) were analyzed for Cloudy Pass, Pine Ridge, HA, and nonHA samples. In the samples from Pine Ridge, a total of 25 OTUs from seven phyla were found to be the core microbiome; in Cloudy Pass there were 387 OTUs including 11 phyla. In comparison, the HA core microbiome (Pine Ridge only) was 46 OTUs representing eight phyla and the non-HA core microbiome (Pine Ridge only) was 479 OTUs representing 14 phyla. Phyla representing the most highly abundant taxa in the Pine Ridge core rhizomicrobiome were Crenarchaeota (Archaea), Acidobacteria, and Actinobacteria, respectively (Fig. 5). The core microbiome from Cloudy Pass, however, did not include Archaea, and had Acidobacteria, Proteobacteria, and Bacteroidetes as its most abundant phyla (Fig. 5). The HA core microbiome included Acidobacteria, Crenarchaeota (Archaea), and Proteobacteria (including Devosia) as its major phyla (Fig. 6). The non-HA (PR) core microbiome included several Archaeal taxa, but included Acidobacteria, Actinobacteria, and Bacteroidetes as its major phyla (Fig. 6).

Culture-based studies. Se tolerance of bacterial isolates from seleniferous and nonseleniferous habitats. When the Cloudy Pass and Pine Ridge rhizosphere and bulk soil samples were cultivated on standardized agar medium at $30^{\circ} \mathrm{C}$, a total of 174 different isolates were obtained, based on morphology and MALDI-TOF, MALDI Biotyper identification (Supplementary Table S2). The isolate numbers for each category (site, host species) are not directly comparable, since the amount of soil used from each sample for bacterial isolation was not standardized.

The isolates were then streaked onto agar plates spiked with different concentrations up to $200 \mathrm{mM}$ selenite or selenate, to test for Se resistance and for the ability to produce red elemental Se

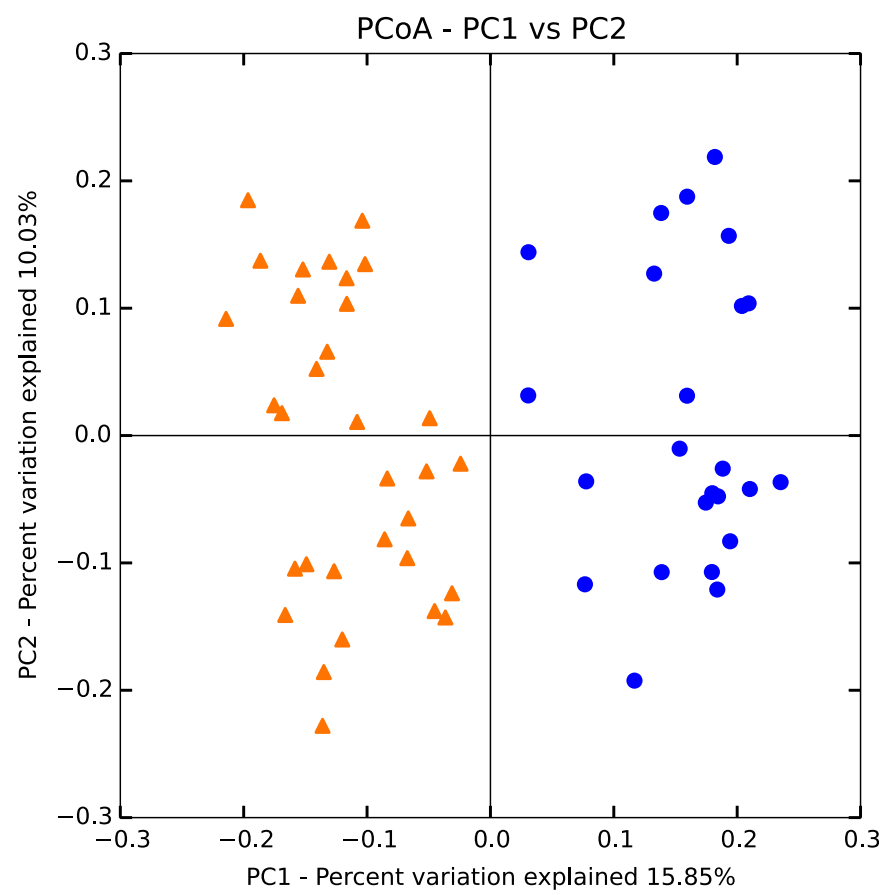

Fig. 2. Two-dimensional principal coordinates analysis ( $\mathrm{PCoA})$ plots of 16S rRNA diversity from rhizosphere soil samples. Orange triangles represent samples from seleniferous site Pine Ridge and blue circles represent samples from nonseleniferous site Cloudy Pass. Permanova test indicates microbial communities are significantly different based on site $(P<0.0001)$.
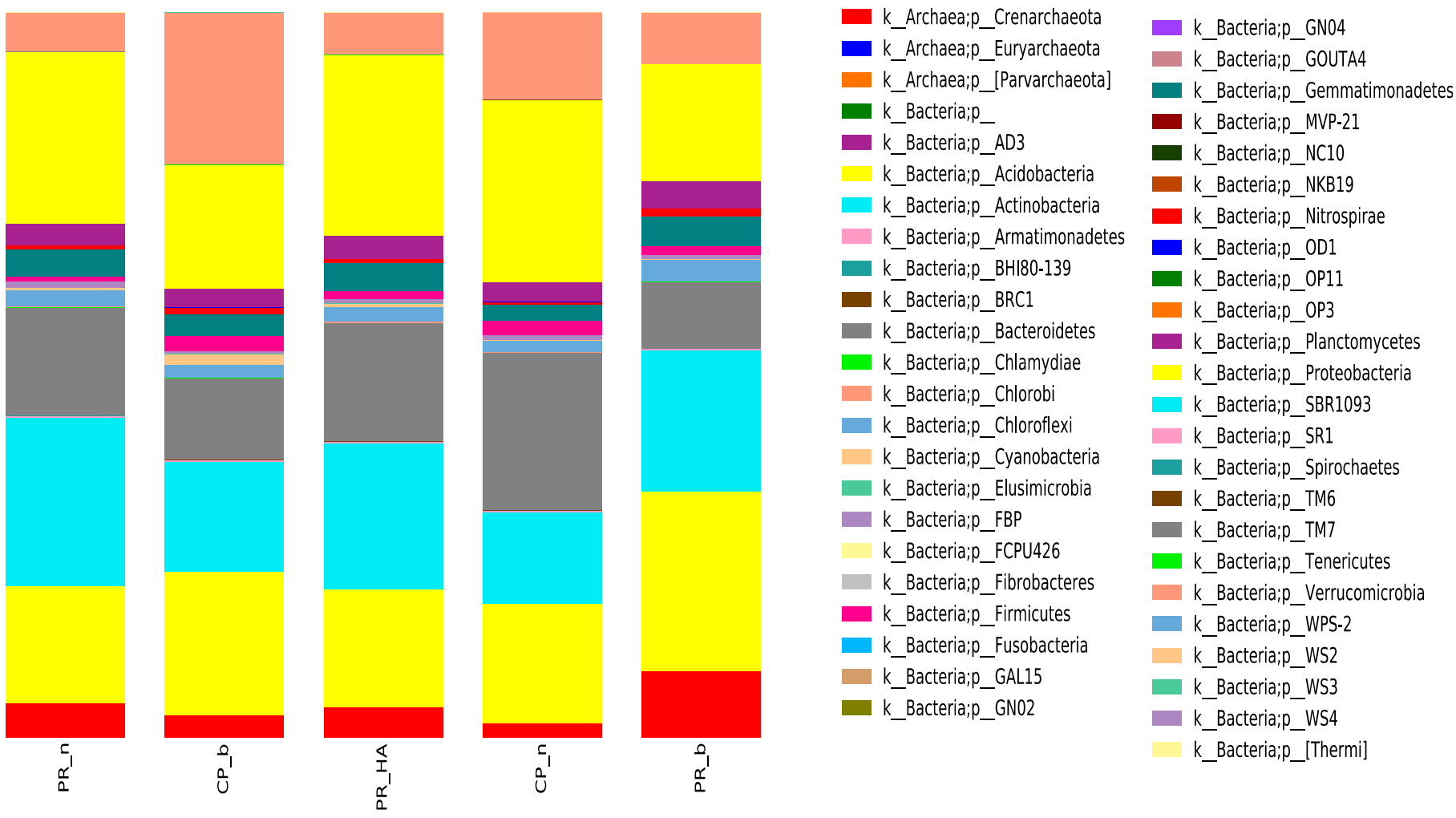

Fig. 1. Chart depicting the relative abundance of bacterial phyla in soil samples from seleniferous (Pine Ridge) and nonseleniferous (Cloudy Pass) sites. From left to right: Pine Ridge non-hyperaccumulator rhizosphere (PR_n), Cloudy Pass bulk soil (CP_b), Pine Ridge hyperaccumulator rhizosphere (PR_HA), Cloudy Pass non-hyperaccumulator rhizosphere (CP_n), and Pine Ridge bulk soil (PR_b). Operational taxonomic unit (OTU) IDs were assigned with QIIME using closed OTU picking against a Green Genes database. Exact percentages out of $100 \%$ of phyla represented in each sampling category is shown in Supplementary Table $\mathrm{S} 1$. 
$\left(\mathrm{Se}^{0}\right)$. To put this in HA context, soil around HA plants has been found to contain up to about $100 \mathrm{mg}$ of $\mathrm{Se} \mathrm{kg}^{-1}$ of soil (El Mehdawi et al. 2011a,b), or $\sim 1.3 \mathrm{mM} \mathrm{Se}$, and the plant tissue itself may contain up to $\sim 2,000 \mathrm{mg}$ of $\mathrm{Se} \mathrm{kg}^{-1}$ of FW $\left(10,000 \mathrm{mg}\right.$ of $\mathrm{Se} \mathrm{kg}^{-1}$ of DW), which corresponds with about $26 \mathrm{mM}$ Se, if evenly distributed. Thus, in nature Se levels of $200 \mathrm{mM}$ are not likely to be encountered by microbes. The highest concentrations were included because in earlier studies endophytic bacteria isolated from these same HA species were found to be resistant up to $200 \mathrm{mM} \mathrm{Se}$ (Sura-de Jong et al. 2015). Qualitative resistance scores were assigned to each isolate based on its ability to grow on certain concentrations of Se. Red Se was observed for all isolates when grown on selenite, but not selenate. The median tolerance scores for the bacteria isolated from each host at each site are represented in Figures 7 and 8. A Kruskal-Wallis test was performed on the median selenite $\left(\mathrm{SeO}_{3}{ }^{2-}\right)$ and selenate $\left(\mathrm{SeO}_{4}{ }^{2-}\right)$ tolerance scores of the pooled isolates from each host plant. No significant differences were found between Se tolerance and host plant species, site, host Se concentration, or bacterial genus. All bacteria isolated from rhizosphere samples were tolerant to most concentrations of $\mathrm{SeO}_{4}{ }^{2-}$. A few isolates even appeared to grow better in the presence of $200 \mathrm{mM} \mathrm{SeO}_{4}{ }^{2-}$ as compared with control conditions, although it cannot be excluded that the larger colony size was due to a matrix of extracellular polymeric substances, produced as a detoxification mechanism. Most isolates were able to grow on the $10 \mathrm{mM} \mathrm{NaSeO}{ }_{3}{ }^{2-}$ plates; however, few strains grew on the $200 \mathrm{mM} \mathrm{NaSeO}{ }^{2-}$ plates.

\section{DISCUSSION}

The main findings from this study are as follows: (i) bacterial and archaeal communities in the rhizosphere of Se HA plants were significantly different from those of non-HA plants growing on the same seleniferous soil and from bulk soil from the same site; (ii)

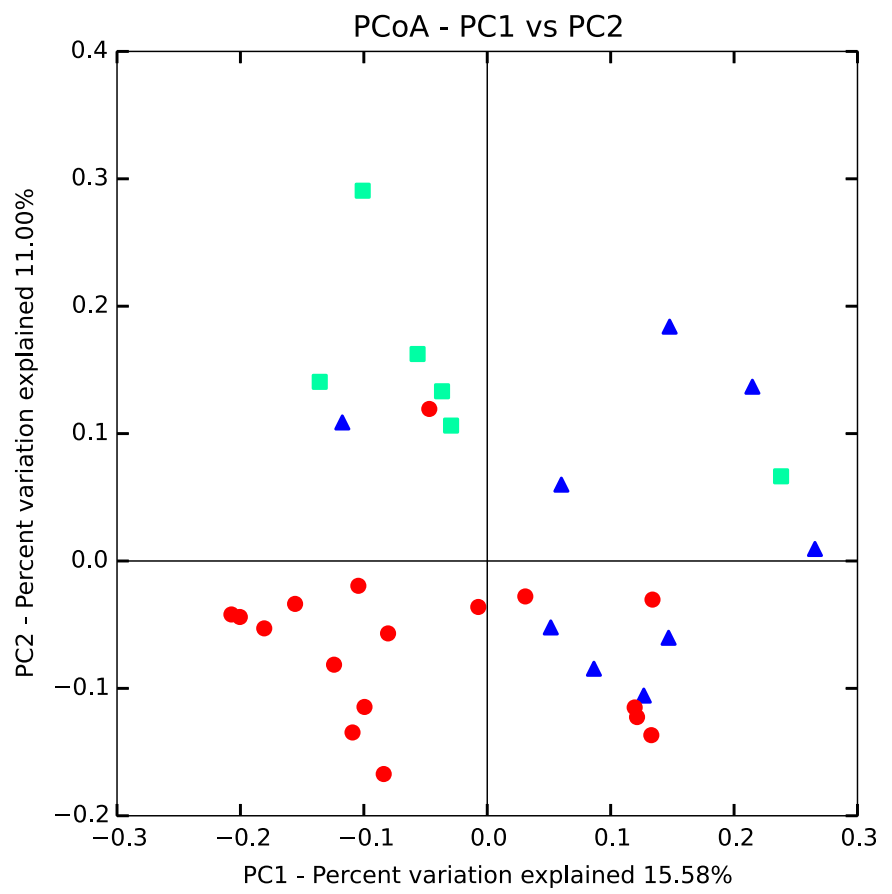

Fig. 3. Two-dimensional principal coordinates analysis (PCoA) plots of 16S rRNA diversity from soil samples collected from seleniferous site Pine Ridge. Red circles are samples taken from selenium hyperaccumulator hosts, blue triangles are non-hyperaccumulator hosts, and green squares are from bulk soil. Permanova test indicates microbial communities are significantly different between the groups $(P<0.001)$. the rhizosphere microbiomes of Se HAs were at least as diverse as those of non-HAs from the same site, and species richness was actually higher; (iii) bacterial and archaeal communities of the rhizosphere differed between the seleniferous and nonseleniferous site; and (iv) most bacterial isolates were extremely selenate- and selenite-resistant (up to $200 \mathrm{mM}$ ), with no clear difference in Se resistance between bacteria isolated from HA and non-HAs or from seleniferous and nonseleniferous soils. Together these results suggest that Se hyperaccumulation can significantly affect a plant's microbiome. In future studies it will be interesting to explore whether these results, obtained from a modest set of data (one seleniferous and one nonseleniferous site, and three HA versus two to three non-HA species, $n=6$ ) will hold true across larger datasets.

These results provide some insight into evolutionary and ecological processes associated with hyperaccumulator-microbiota interactions. The finding that the HA rhizosphere microbial composition was significantly different from the non-HA rhizosphere samples on the same seleniferous site may indicate that HAs select for, or allow specific bacterial communities to colonize their rhizosphere. The finding that three HA plant species from different families differed significantly in rhizosphere microbiome from two non-HA plant species from the same families suggests Se HAs have a common rhizosphere factor that affects rhizomicrobial composition (Fig. 3). This common factor is not necessarily Se-related, but it may well be. Judged from the Se resistance assays, Se resistance likely is not under direct selection in the rhizosphere because all rhizosphere bacterial isolates were resistant to Se levels beyond those commonly found in the rhizosphere of HA (as discussed in
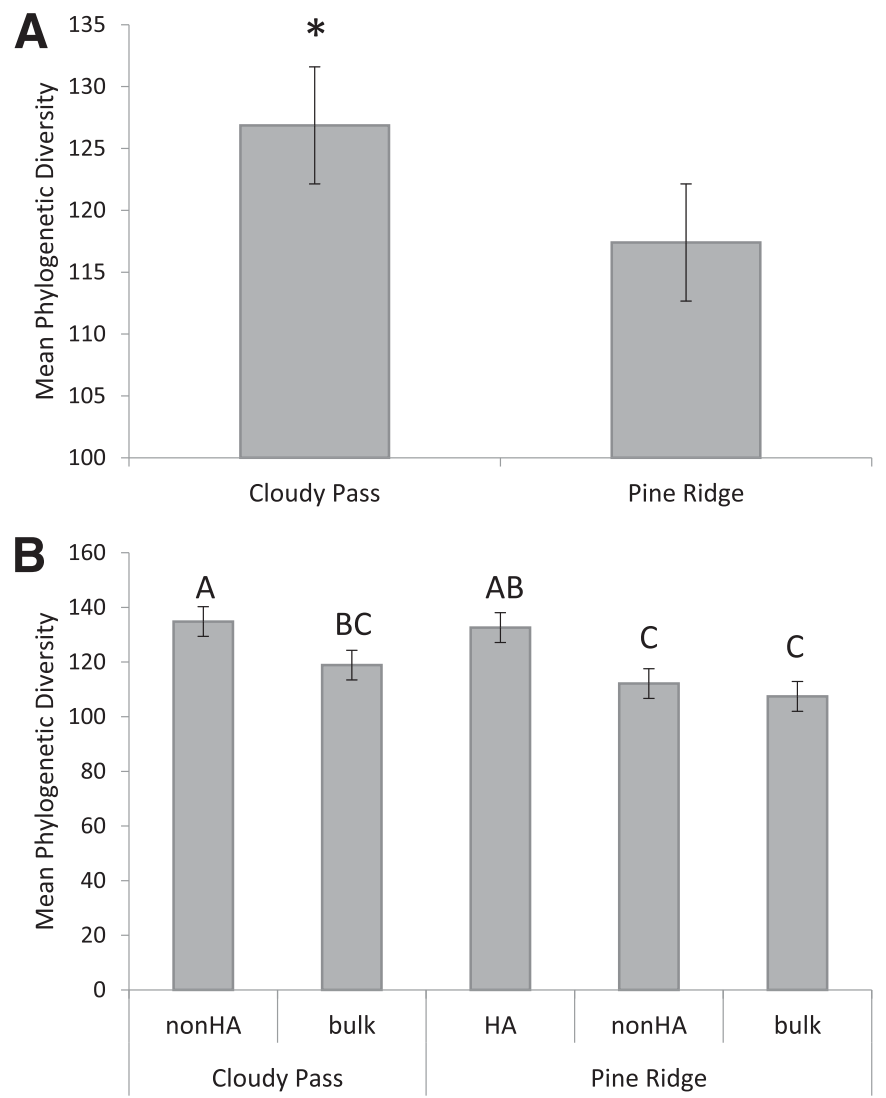

Fig. 4. Alpha diversity (observed operational taxonomic units) of soil samples in A, each site and $\mathbf{B}$, each group. Analysis of variance was performed on each data set and both were found to be statistically significant (A, $P<0.004 ; \mathbf{B}, P<0.0001)$. Letters in $\mathbf{B}$ designated by Tukey's honest significant difference indicate significant differences. 
more detail below). However, the difference in bacterial composition between HA and non-HA plants may still be Se-related. Hyperaccumulators were found to have high Se levels in their rhizosphere (around $100 \mathrm{mg}$ of $\mathrm{Se} \mathrm{kg}^{-1}$ of soil, among the highest soil Se levels reported anywhere), mostly in organic (C-Se-C) forms similar to the main form found in Se HA plants (El Mehdawi et al. 2015). Some bacteria may be better able than others to utilize this organic Se and thus benefit from it in terms of fitness. Such microbes would have a higher fitness in the rhizosphere of Se HAs. Two genera of interest, in this respect, are Pedobacter and Devosia. These genera showed the highest average occurrence in HAs (when compared with non-HA and bulk soil on Pine Ridge samples) and Devosia was also part of the core microbiome of the Se HAs, as part of about 25 total taxa present in $100 \%$ of the HA samples. Even though our culture-independent analyses showed these two genera to be of interest, neither Pedobacter nor Devosia were isolated using culture-based techniques.

While the differences in rhizosphere microbial communities between HA and non-HA may reflect the effects of Se HA, conversely it may be hypothesized that the different, HA-specific rhizobial communities could be one of the factors contributing to Se hyperaccumulation. Establishing symbioses with Se-resistant bacteria that reduce $\mathrm{Se}$ oxyanions to less toxic elemental Se could function as a plant Se resistance mechanism when growing in soils with Se hotspots. It is also feasible that some of the core microbiome taxa facilitate plant Se accumulation, as has been found before in plant inoculation studies using rhizosphere bacteria (as reviewed in the introduction), and thus may contribute to hyperaccumulation.
Indeed, in a study by Alford et al. (2014), root colonization by Rhizobia sp. was correlated with significantly enhanced Se accumulation in HA Astragalus species, especially in the organic form $\gamma$-glutamyl-methylselenocysteine. More studies are needed to determine whether and how the unique HA rhizomicrobiome contributes to plant fitness.

The observed higher species richness in the rhizosphere of Se HAs compared with non-HA at Pine Ridge may in part be explained by seleno-amino acids released by Se HAs via litter and roots, providing more diverse carbon sources and an extra source of essential C, N, and Se to the bacteria (El Mehdawi et al. 2015). Some nonheterotrophic bacteria may also benefit from symbiosis with HA plant roots by utilizing the associated selenocompounds for the dissimilatory use of Se, an ancient form of energy generation in bacteria and archaea by means of anaerobic respiration. The finding that high-Se habitats are rich in microbial diversity is in agreement with earlier studies that showed a high bacterial diversity in Se contaminated areas; for example, in Se contaminated ponds (de Souza et al. 2001), and enhanced numbers of cultivable microbes in Se-rich leaf litter (Quinn et al. 2011a). Similarly, it was found that Ni HA Thlaspi (reclassified as Noccaea) caerulescens also harbored a rhizosphere rich in microbial diversity with Ni-resistant bacteria (Aboudrar et al. 2007). However, several other studies that assessed the species richness of microbial communities in soils containing high concentrations of other elements including $\mathrm{Cr}, \mathrm{Cu}$, and As have shown an opposite effect, where microbial diversity decreases in these conditions (Kong et al. 2006; Sheik et al. 2012). A potential explanation may be that these other

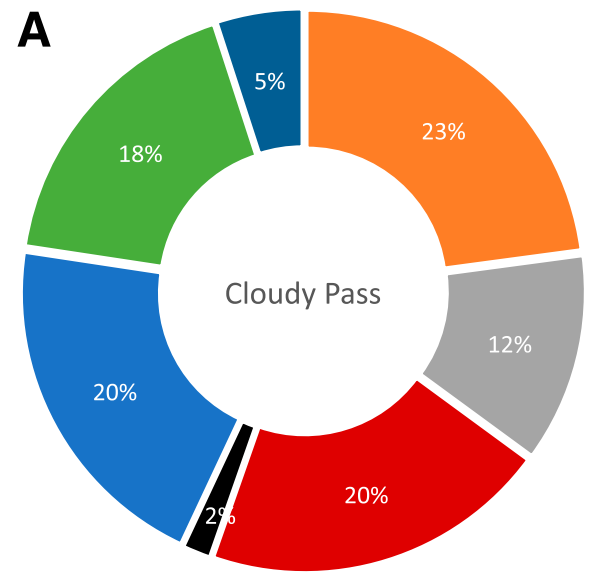

- Acidobacteria

- Actinobacteria

- Bacteroidetes

- Plantcomycetes

- Proteobacteria

- Verrucromicrobia

- Other

B

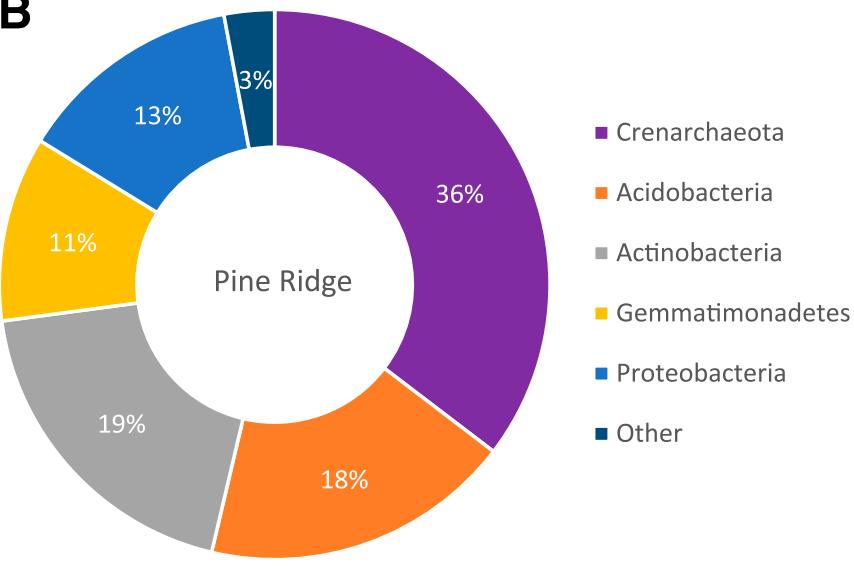

Fig. 5. Pie charts representing the core microbiome (100\%; phylum) of $\mathbf{A}$, Cloudy Pass and B, Pine Ridge.

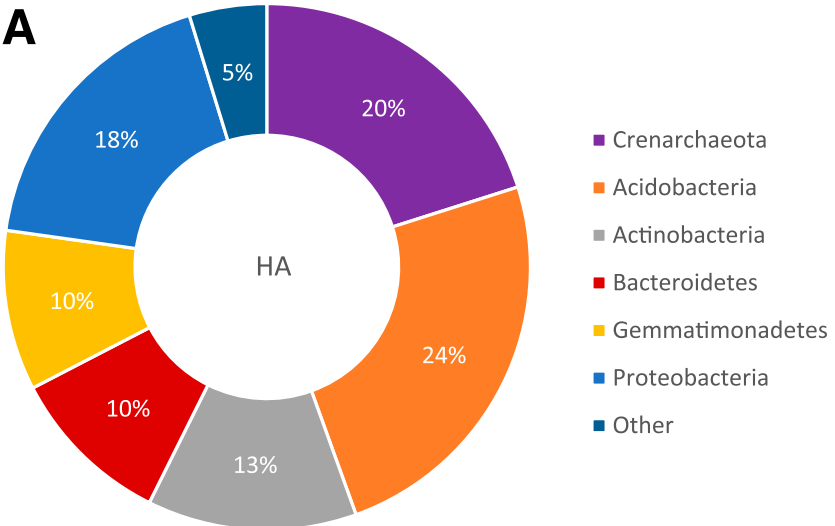

B

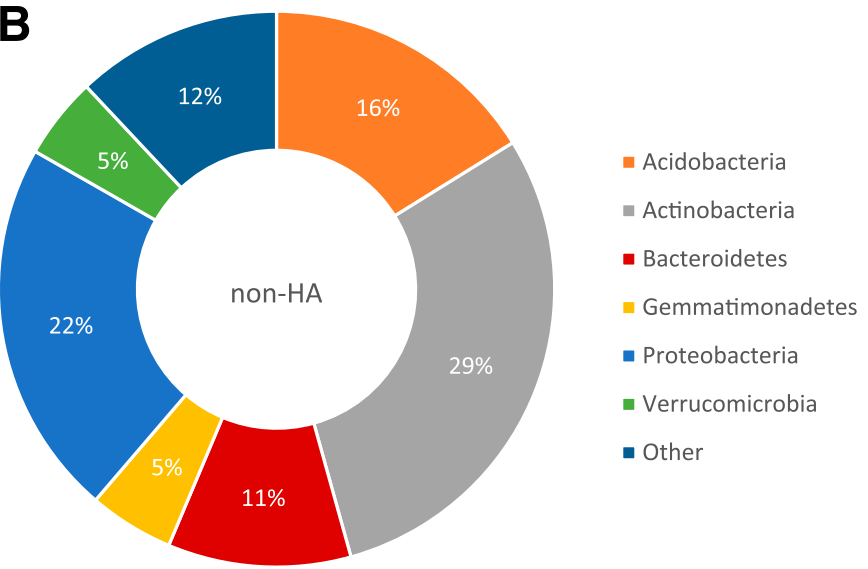

Fig. 6. Pie charts representing the core microbiome at Pine Ridge (100\%; phylum) of $\mathbf{A}$, hyperaccumulators $(\mathrm{HA})$ and $\mathbf{B}$, non-hyperaccumulators (non-HA). 
elements are not present in organic forms or cannot be utilized by bacteria as essential elements.

The rhizosphere microbiome sequencing data revealed that the bacterial communities on the seleniferous and nonseleniferous sites were significantly different. This site-dependent difference
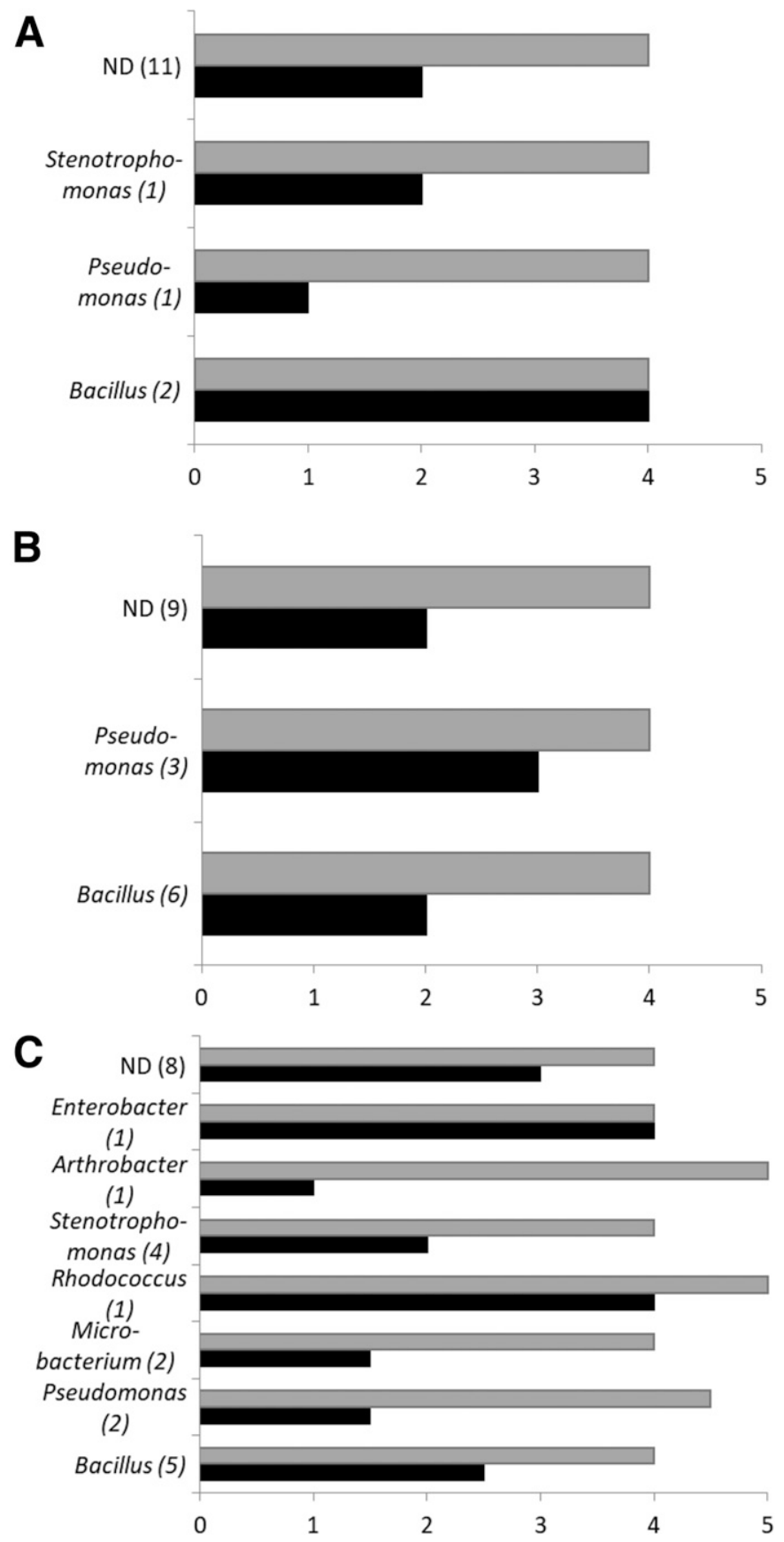

Fig. 7. Median selenite (black) and selenate (gray) resistance scores for bacterial genera isolated from rhizosphere soil samples of different nonhyperaccumulator host plants from nonseleniferous area Cloudy Pass. A, Symphyotrichum ericoides; B, Physaria montana; and C, Astragalus laxmannii. Unidentified bacteria are pooled into not determined (ND). The number of isolates in each genus are in parentheses next to the genus name on the $y$-axis. Tolerance scores were assigned by the following criteria: 0 , no growth $\geq 1 \mathrm{mM} \mathrm{Se}$; , no growth $\geq 10 \mathrm{mM} \mathrm{Se}$; 2 , no growth $\geq 100 \mathrm{mM} \mathrm{Se}$; 3 , no growth $\geq 200 \mathrm{mM} \mathrm{Se}$;, growth at $200 \mathrm{mM}$ Se; and 5, enhanced growth on $200 \mathrm{mM}$ Se relative to $0 \mathrm{Se}$. All isolates grew on 0 Se medium. has been shown before and thus was an expected result (Berg and Smalla 2009). It may be attributed to a range of factors that differed between the two sites, including the soil type, $\mathrm{pH}$, or possibly $\mathrm{Se}$ level.

Bacterial strains associated with plants that HA metals such as $\mathrm{Ni}, \mathrm{Cd}$, and $\mathrm{Zn}$ have been shown to be more tolerant to the metal being accumulated than similar bacteria taken from nonaccumulators (Visioli et al. 2015). This study, however, does not provide evidence that rhizobacteria isolated from Se HAs are more tolerant to Se than rhizobacteria associated with nonaccumulators from either the seleniferous or nonseleniferous site. Rather, all bacterial isolates were highly Se resistant $(>10 \mathrm{mM}$ selenite and $>200 \mathrm{mM}$ selenate). To date, rhizobacteria tested for Se tolerance have only been tested up to $50 \mathrm{mM}$ (Di Gregorio et al. 2003). This study shows that bacteria isolated from rhizosphere soil can survive, and in some cases show enhanced growth, on concentrations of $200 \mathrm{mM}$ selenate and selenite. A similar trend was noticed in the tolerance of endophytes taken isolated from Se HAs Stanleya pinnata and Astragalus bisulcatus from the same seleniferous site sampled here (Pine Ridge), where the isolates were also able to withstand concentrations of $200 \mathrm{mM} \mathrm{Se}$ (Sura-de Jong et al. 2015). Thus, it appears that bacteria in general are much more Se resistant than fungi, animals or plants. For comparison, an earlier study that tested rhizosphere fungi taken from HAs and non-HAs from the same site showed that fungal strains from the rhizosphere of HAs were no more tolerant to $10 \mathrm{mg}$ of Se liter ${ }^{-1}$ (125 $\mu \mathrm{M}$ selenate) than strains isolated from non-HAs from the same seleniferous site (Wangeline et al. 2011). However, in the same study rhizospheric fungi from seleniferous sites were significantly more Se tolerant than those from a nonseleniferous site (Wangeline et al. 2011).

In an earlier study, the Se levels found in soil surrounding these Se HA species were 7 to 11 times elevated compared with bulk soil, but the soil Se levels were never more than a few hundred milligrams per kilogram (El Mehdawi et al. 2011a,b, 2012), which corresponds with a few millimolar selenate or selenite. Most isolates in this study, both from seleniferous and nonseleniferous soil, were not inhibited by a few millimolar selenate or selenite. This suggests that most bacteria are not sensitive to the concentrations of Se that typically surround $\mathrm{Se}$ HAs (the site studied here is typical for seleniferous sites in the United States), and that the bacterial microbiome in the rhizosphere of HAs is not under selection for Se resistance. This result is quite different from those found for other ecological partners associated with HAs: herbivores, fungi, other plant species, and also pollinators are generally sensitive to the high levels of Se in HAs (El Mehdawi et al. 2011a,b, 2012, 2015; Freeman et al. 2007, 2009; Galeas et al. 2008; Hanson et al. 2003, 2004; PilonSmits 2012a,b, 2015; Quinn et al. 2008, 2011b; Valdez Barillas et al. 2012). This general Se sensitivity has been suggested to lead to a selective effect by Se HAs on their ecological partners, against Se sensitivity and for Se resistance (El Mehdawi and Pilon-Smits 2012). Thus, while Se HAs may be hypothesized to significantly affect the Se resistance and perhaps through that, species composition of their associated herbivores, pollinators, and vegetation, they likely do not directly select for enhanced Se resistance in their microbiome. Nevertheless, the organic selenocompounds present in HA-associated habitats may affect microbial species composition, favoring taxa that best utilize the essential element Se. Hyperaccumulator rhizosphere microbiomes showed increased species richness and significantly different community composition. More studies are needed to investigate the nature of the relationships between Se HAs and their specific bacterial microbiome. 
From an applied perspective, the findings described here are of interest because some of the bacterial taxa found in the core rhizosphere microbiome of Se hyperaccumulators may have properties that make them suitable to be utilized for bioremediation of Se polluted soils and waterways or for Se biofortification of crops or phytoremediation. These applications have broad significance: Se deficiency has been estimated to affect a billion people worldwide, and Se toxicity affects hundreds of millions (Schiavon and Pilon-Smits 2017).
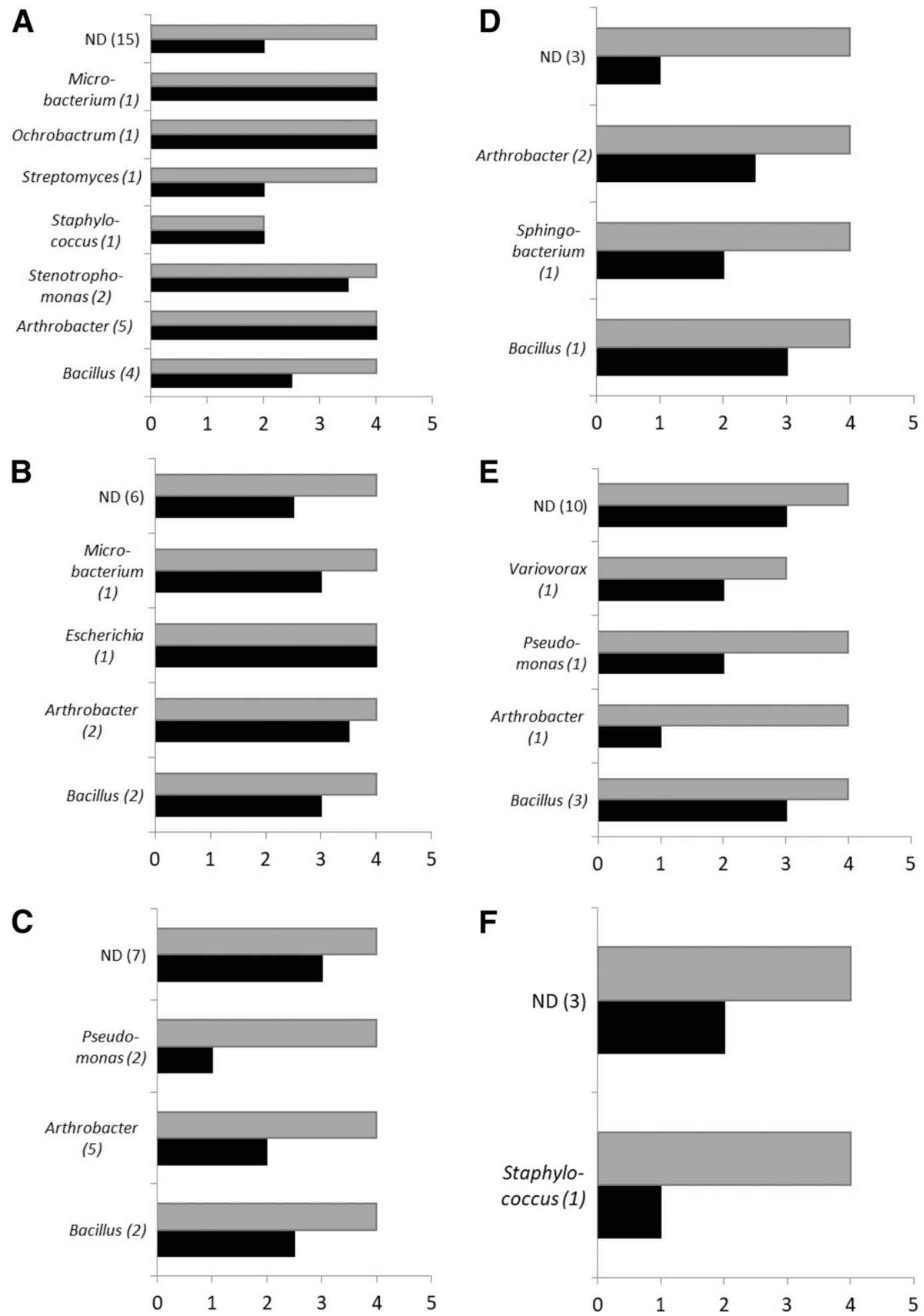

Fig. 8. Median selenite (black) and selenate (gray) tolerance scores for each bacterial genus isolated from rhizosphere soil samples of different host species (or bulk soil) from seleniferous area Pine Ridge. Isolates from hyperaccumulator hosts are on the left (A, Astragalus bisulcatus; B, Stanleya pinnata; and C, Symphyotrichum ericoides). Isolates from non-hyperaccumulator hosts are on the right (D, Astragalus tenellus; E, Physaria bellii; and F, bulk soil). Unidentified bacteria are pooled into category not determined (ND). The number of isolates in each genus are shown in parentheses next to the genus names on the $y$-axis. Tolerance scores were assigned by the following criteria: 0 , no growth $\geq 1 \mathrm{mM} \mathrm{Se} ; 1$, no growth $\geq 10 \mathrm{mM} \mathrm{Se} ; 2$, no growth $\geq 100 \mathrm{mM}$ Se; 3 , no growth $\geq 200 \mathrm{mM} \mathrm{Se}$;, growth at $200 \mathrm{mM} \mathrm{Se}$; and 5, enhanced growth on $200 \mathrm{mM}$ Se relative to 0 Se. All isolates grew on 0 Se medium. 


\section{ACKNOWLEDGMENTS}

Data processing, 16S rRNA sequencing, and core amplicon data analysis were performed by the Earth Microbiome Project (www. earthmicrobiome.org), and all amplicon sequence data and metadata have been made public through the data portal (http://qiita. ucsd.edu). Identification of isolates was possible thanks to the project KONTAKT LH12087 of the Czech Ministry of Education, Youth and Sports. We thank J. Leach, M. Stromberger, and J. Von Fischer for providing helpful comments towards this study.

\section{LITERATURE CITED}

Aboudrar, W., Schwartz, C., Benizri, E., Morel, J. L., and Boularbah, A. 2007. Soil microbial diversity as affected by the rhizosphere of the hyperaccumulator Thlaspi caerulescens under natural conditions. Int. J. Phytoremediation 9:41-52.

Alford, E. R., Lindblom, S. D., Pittarello, M., Freeman, J. L., Fakra, S. C., Marcus, M. A., Broeckling, C., Pilon-Smits, E. A. H., and Paschke, M. W. 2014. Roles of rhizobial symbionts in selenium accumulation in Astragalus (Fabaceae). Am. J. Bot. 101:1895-1905.

Alford, E. R., Pilon-Smits, E. A. H., and Paschke, M. W. 2010. Metallophytes-A view from the rhizosphere. Plant Soil 337:33-50.

Baker, A. J. M., and Brooks, R. R. 1989. Terrestrial higher plants with hyperaccumulate metallic elements-A review of their distribution, ecology and phytochemistry. Biorecovery 1:81-126.

Beath, O. A., Gilbert, C. S., and Eppson, H. F. 1939. The use of indicator plants in locating seleniferous areas in Western United States. I. General. Am. J. Bot. 26:257-269.

Berg, G., and Hallmann, J. 2006. Control of plant pathogenic fungi with bacterial endophytes. Pages 53-69 in: Microbial Root Endophytes Part I. B. J. E. Schulz, C. J. C. Boyle, and T. N. Seiber, eds. Springer, Berlin, Heidelberg.

Berg, G., and Smalla, K. 2009. Plant species and soil type cooperatively shape the structure and function of microbial communities in the rhizosphere. F.E.M.S. Microbiol. Ecol. 68:1-13.

Berg, G., Zachow, C., Müller, H., Phillips, J., and Tilcher, R. 2013. Nextgeneration bio-products sowing the seeds of success for sustainable agriculture. Agronomy (Basel) 3:648-656.

Caporaso, J. G., Kuczynski, J., Stombaugh, J., Bittinger, K., Bushman, F. D., Costello, E. K., Fierer, N., Gonzalez Pena, A., Goodrich, J. K., Gordon, J. I., Huttley, G. A., Kelley, S. T., Knights, D., Koenig, J. E., Ley, R. E., Lozupone, C. A., McDonald, D., Muegge, B. D., Pirrung, M., Reeder, J., Sevinsky, J. R., Turnbaugh, P. J., Walters, W. A., Widmann, J., Yatsunenko, T., Zaneveld, J., and Knight, R. 2010. QIIME allows analysis of high-throughput community sequencing data. Nat. Methods 7:335-336.

Cappa, J. J., and Pilon-Smits, E. A. H. 2014. Evolutionary aspects of elemental hyperaccumulation. Planta 239:267-275.

de Souza, M. P., Amini, A., Dojka, M. A., Pickering, I. J., Dawson, S. C., Pace, N. R., and Terry, N. 2001. Identification and characterization of bacteria in a selenium-contaminated hypersaline evaporation pond. Appl. Environ. Microbiol. 67:3785-3794.

de Souza, M. P., Chu, D., Zhao, M., Zayed, A. M., Ruzin, S. E., Schichnes, D., and Terry, N. 1999a. Rhizosphere bacteria enhance selenium accumulation and volatilization by Indian mustard. Plant Physiol. 119:565-573.

de Souza, M. P., Huang, C. P. A., Chee, N., and Terry, N. 1999b. Rhizosphere bacteria enhance the accumulation of selenium and mercury in wetland plants. Planta 209:259-263.

Di Gregorio, S., Lampis, S., Paganella, M., and Vallini, G. 2003. Reduction of selenite to elemental selenium by a bacterial strain of Stenotrophomonas sp. isolated from the rhizosphere of the hyperaccumulator legume Astragalus bisulcatus. Page F06 in: In situ and on-site bioremediation. V. S. Magar and M. E. Kelley, eds. Proceedings of the Seventh International In Situ and OnSite Bioremediation Symposium, Florida.

Di Gregorio, S., Lampis, S., and Vallini, G. 2005. Selenite precipitation by a rhizospheric strain of Stenotrophomonas sp. isolated from the root system of Astragalus bisulcatus: A biotechnological perspective. Environ. Int. 31: 233-241.

El Mehdawi, A. F., Cappa, J. J., Fakra, S. C., Self, J., and Pilon-Smits, E. A. H. 2012. Interactions of selenium hyperaccumulators and nonaccumulators during cocultivation on seleniferous or nonseleniferous soil- the importance of having good neighbors. New Phytol. 194:264-277.
El Mehdawi, A. F., Paschke, M., and Pilon-Smits, E. A. H. 2015. Symphyotrichum ericoides populations from seleniferous and nonseleniferous soil display striking variation in selenium accumulation. New Phytol. 206:231-242.

El Mehdawi, A. F., and Pilon-Smits, E. A. H. 2012. Ecological aspects of plant selenium hyperaccumulation. Plant Biol. 14:1-10.

El Mehdawi, A. F., Quinn, C. F., and Pilon-Smits, E. A. H. 2011a. Effects of selenium hyperaccumulation on plant-plant interactions: Evidence for elemental allelopathy? New Phytol. 191:120-131.

El Mehdawi, A. F., Quinn, C. F., and Pilon-Smits, E. A. H. 2011b. Selenium hyperaccumulators facilitate selenium-tolerant neighbors via phytoenrichment and reduced herbivory. Curr. Biol. 21:1440-1449.

Evans, C. J. A. C. S., and Johnson, C. M. 1967. Collection and partial characterization of volatile selenium compounds from Medicago sativa L. Aust. J. Biol. Sci. 20:737-748.

Fassel, V. A. 1978. Quantitative elemental analyses by plasma emission spectroscopy. Science 202:183-191.

Frankenberger, W. T., and Karlson, U. 1994. Microbial Volatilization of selenium from soils and sediments. Pages 369-387 in: Selenium in the Environment. W. T. Frankenberger Jr. and S. Benson, eds. Marcel Dekker, New York.

Freeman, J. L., Lindblom, S. D., Quinn, C. F., Fakra, S., Marcus, M. A., and Pilon-Smits, E. A. H. 2007. Selenium accumulation protects plants from herbivory by orthoptera due to toxicity and deterrence. New Phytol. 175: 490-500.

Freeman, J. L., Quinn, C. F., Lindblom, S. D., Klamper, E. M., and Pilon-Smits, E. A. H. 2009. Selenium protects the hyperaccumulator Stanleya pinnata against black-tailed prairie dog herbivory in native seleniferous habitats. Am. J. Bot. 96:1075-1085.

Galeas, M. L., Klamper, E. M., Bennett, L. E., Freeman, J. L., Kondratieff, B. C., and Pilon-Smits, E. A. H. 2008. Selenium hyperaccumulation reduces plant arthropod loads in the field. New Phytol. 177:715-724.

Garbisu, C., Ishii, T., Leighton, T., and Buchanan, B. B. 1996. Bacterial reduction of selenite to elemental selenium. Chem. Geol. 132:199-204.

Gilbert, J. A., Jansson, J. K., and Knight, R. 2014. The Earth Microbiome project: Successes and aspirations. BMC Biol. 12:69.

Hanson, B., Lindblom, S. D., Garifullina, G. F., Wangeline, A., Ackley, A., and Pilon-Smits, E. A. H. 2003. Selenium accumulation protects Brassica juncea susceptibility to invertebrate herbivory and fungal infection. New Phytol. 159:461-469.

Hanson, B. R., Lindblom, S. D., Loeffler, M. L., and Pilon-Smits, E. A. H. 2004. Selenium protects plants from phloem-feeding aphids due to both deterrence and toxicity. New Phytol. 162:655-662.

Kong, W. D., Zhu, Y. G., Fu, B. J., Marschner, P., and He, J. Z. 2006. The veterinary antibiotic oxytetracycline and $\mathrm{Cu}$ influence functional diversity of the soil microbial community. Environ. Pollut. 143:129-137.

Lindblom, S. D., Fakra, S. C., Landon, J., Schulz, P., Tracy, B., and Pilon-Smits, E. A. H. 2012. Inoculation of Astragalus racemosus and Astragalus convallarius with selenium-hyperaccumulator rhizosphere fungi affects growth and selenium accumulation. Planta 237:717-729.

Lindblom, S. D., Fakra, S. C., Landon, J., Schulz, P., Tracy, B., and Pilon-Smits, E. A. H. 2013. Inoculation of selenium hyperaccumulator Stanleya pinnata and related non-accumulator Stanleya elata with hyperaccumulator rhizosphere fungi-Investigation of effects on Se accumulation and speciation. Physiol. Plant. 150:107-118.

Luo, J., Tao, Q., Wu, K., Li, J., Qian, J., Liang, Y., Yang, X., and Li, T. 2017. Structural and functional variability in root-associated bacterial microbiomes of $\mathrm{Cd} / \mathrm{Zn}$ hyperaccumulator Sedum alfredii. Appl. Microbiol. Biotechnol. 101:7961-7976.

Neuhierl, B., Thanbichler, M., Lottspeich, F., and Böck, A. 1999. A family of S-methylmethionine-dependent thiol/selenol methyltransferases. Role in selenium tolerance and evolutionary relation. J. Biol. Chem. 274: 5407-5414.

Pilon-Smits, E. A. H. 2012a. Plant accumulation of sulfur's sister element selenium-Potential applications and ecological implications. Pages 145153 in: Sulfur Metabolism in Plants: Mechanisms and Application to Food Security, and Responses to Climate Change. L. J. de Kok, M. Tausz, M. J. Hawkesford, R. Hoefgen, T. McManus, R. M. Norton, H. Rennenberg, K. Saito, E. Schnug, and L. Tabe, eds. Springer, Dordrecht, the Netherlands.

Pilon-Smits, E. A. H. 2012b. Plant selenium metabolism; Genetic manipulation, phytotechnological applications and ecological implications. Pages 293-311 in: Environmental Contamination-Health Risks, Bioavailability and Bioremediation. M. H. Wong, ed. Taylor and Francis. 
Pilon-Smits, E. A. H. 2015. Selenium in plants. Pages 93-107 in: Progress in Botany. Vol. 76. U. Luttge and W. Beyschlag, eds. Springer International Publishing, Switzerland.

Pilon-Smits, E. A. H., Quinn, C. F., Tapken, W., Malagoli, M., and Schivaon, M. 2009. Physiological functions of beneficial elements. 94th E.S.A. Annual Meeting.

Quinn, C. F., Freeman, J. F., Galeas, M. L., Klamper, E. M., and Pilon-Smits, E. A. H. 2008. The role of selenium in protecting plants against prairie dog herbivory: Implications for the evolution of selenium hyperaccumulation. Oecologia 155:267-275.

Quinn, C. F., Prins, C. N., Gross, A. M., Hantzis, L., Reynolds, R. J. B., Freeman, J. L., Yang, S. I., Covy, P. A., Bañuelos, G. S., Pickering, I. J., Fakra, S. F., Marcus, M. A., Arathi, H. S., and Pilon-Smits, E. A. H. 2011b. Selenium accumulation in flowers and its effects on pollination. New Phytol. 192:727-737.

Quinn, C. F., Wyant, K., Wangeline, A. L., Shulman, J., Galeas, M. L., Valdez, J. R., Paschke, M. W., and Pilon-Smits, E. A. H. 2011a. Selenium hyperaccumulation increases leaf decomposition rate in a seleniferous habitat-Evidence for specialist decomposers? Plant Soil 341:51-61.

Schiavon, M., and Pilon-Smits, E. A. H. 2017. The fascinating facets of plant selenium accumulation-Biochemistry, physiology, evolution and ecology. New Phytol. 213:1582-1596.

Shade, A., and Handelsman, J. 2011. Beyond the Venn diagram: The hunt for a core microbiome. Environ. Microbiol. 14:4-12.

Sheik, C. S., Mitchell, T. W., Rizvi, F. Z., Rehman, Y., Faisal, M., Hasnain, S., McInerney, M. J., and Krumholz, L. R. 2012. Exposure of soil microbial communities to chromium and arsenic alters their diversity and structure. PLoS One 7:e40059.

Staicu, L. C., Ackerson, C. J., Cornelis, P., Ye, L., Berendsen, R. L., Hunter, W. J., Noblitt, S. D., Henry, C. S., Cappa, J. J., Montenieri, R. L., Wong, A. O., Musilova, L., Sura-de Jong, M., van Hullebusch, E. D., Lens, P. N. L., Reynolds, R. J. B., and Pilon-Smits, E. A. H. 2015b. Pseudomonas moraviensis subsp. stanleyae, a bacterial endophyte of hyperaccumulator Stanleya pinnata, is capable of efficient selenite reduction to elemental selenium under aerobic conditions. J. Appl. Microbiol. 119:400-410.

Staicu, L. C., van Hullebusch, E. D., Lens, P. N. L., Pilon-Smits, E. A. H., and Oturan, M. A. 2015a. Electrocoagulation of colloidal biogenic selenium. Environ. Sci. Pollut. Res. 22:3127-3137.
Sura-de Jong, M., Reynolds, R. J., Richterova, K., Musilova, L., Hrochova, I., Frantik, T., Sakmaryova, I., Strejcek, M., Cochran, A. T., Staicu, L., Cappa, J. J., van der Lelie, D., and Pilon-Smits, E. A. H. 2015. Selenium hyperaccumulators harbor a diverse endophytic bacterial community characterized by high selenium resistance and plant growth promoting properties. Front. Plant Sci. 6:113.

Terry, N., Zayed, A. M., de Souza, M. P., and Tarun, A. S. 2000. Selenium in higher plants. Annu. Rev. Plant Physiol. Plant Mol. Biol. 51:401-432.

Thompson, L. R., Sanders, J. G., McDonald, D., Amir, A., Ladau, J., Locey, K. J. 2017. A communal catalogue reveals Earth's multiscale microbial diversity. Nature 551:457-463.

Turner, R. J., Weiner, J. H., and Taylor, D. E. 1998. Selenium metabolism in Escherichia coli. Biometals 11:223-227.

Valdez Barillas, J. R., Quinn, C. F., Freeman, J. L., Lindblom, S. D., Marcus, M. S., Fakra, S. C., Gilligan, T. M., Alford, E. R., Wangeline, A. L., and Pilon-Smits, E. A. H. 2012. Selenium distribution and speciation in hyperaccumulator Astragalus bisulcatus and associated ecological partners. Plant Physiol. 159:1834-1844.

Visioli, G., D'Egidio, S., and Sanangelantoni, A. M. 2015. The bacterial rhizobiome of hyperaccumulators: Future perspectives based on omics analysis and advanced microscopy. Front. Plant Sci. 5:752.

Wangeline, A. L., Valdez, J. R., Lindblom, S. D., Bowling, K. L., Reeves, F. B., and Pilon-Smits, E. A. H. 2011. Selenium tolerance in rhizosphere fungi from Se hyperaccumulator and non-hyperaccumulator plants. AMJB 98:11391147.

Winkel, L. H. E., Vriens, B., Jones, G. D., Schneider, L. S., Pilon-Smits, E. A. H., and Banuelos, G. S. 2015. Selenium cycling across soil-plant-atmosphere interfaces: A critical review. Nutrients 7:4199-4239.

Yasin, M., El Mehdawi, A. F., Anwar, A., Pilon-Smits, E. A. H., and Faisal, M. 2015. Microbial-enhanced selenium and iron biofortification of wheat (Triticum aestivum L.)-Applications in phytoremediation and biofortification. Int. J. Phytoremediation 17:341-347.

Zayed, A. M., and Terry, N. 1994. Selenium volatilization in roots and shoots: Effects of shoot removal and sulfate level. J. Plant Physiol. 143:8-14.

Zhang, Y., and Gladyshev, V. N. 2009. Comparative genomic analyses of trace element utilization and evolution. FASEB J. 23:103.8. 